\title{
HARMONIC MAPPINGS AND \\ Hyperbolic PlüCKer Transformations
}

Klaus List

Let $\Pi_{h}$ be a 3-dimensional hyperbolic space with Euclidean ground field $\mathbb{K}$. There is a certain procedure by which any harmonic mapping of the projective line over the unique quadratic extension of $\mathbb{K}$ induces an orthogonality-preserving Plücker transformation of $\Pi_{h}$ and, conversely, any orthogonality-preserving Plücker transformation of $\Pi_{h}$ is induced by such a harmonic mapping.

\section{INTRODUCTION}

In this paper we discuss orthogonality-preserving Plücker transformations of a 3-dimensional hyperbolic space with Euclidean ground field $\mathbb{K}$. They are characterized by the property that two hyperbolic lines intersect orthogonally if and only if their images do so. In fact it is sufficient to demand the if part of this characterization (Proposition 2). In [12] the same Plücker transformations are discussed in hyperbolic planes and hyperbolic spaces with dimension $\geq 4$. It turns out [12] that those Plücker transformations are exactly the bijections of the set of hyperbolic lines that come from collineations of the hyperbolic space. In the Cayley-Klein model, which is based upon an absolute quadric $\mathcal{Q}$, such a "hyperbolic collineation" extends to a unique collineation of the ambient projective space. Conversely, each automorphic collineation of $\mathcal{Q}$ yields a hyperbolic collineation. We will answer the question if all orthogonality-preserving Plücker transformations in hyperbolic 3spaces are also induced by collineations. The parallel classes of the hyperbolic space are in one-one correspondence with the points of the absolute quadric $\mathcal{Q}$ in the Cayley-Klein model. The quadric $\mathcal{Q}$ in turn can be identified with the projective line $\Pi_{\mathbb{L}}$ over the unique quadratic extension $\mathbb{L}$ of $\mathbb{K}$. It turns out that each Plücker transformation preserves the hyperbolic parallelism. Moreover, by the above identification, each Plücker transformation corresponds to a harmonic mapping of $\Pi_{\mathbb{L}}$, and vice versa (Theorem 1).

By the Theorem of StAUdT-HuA each harmonic mapping can be decomposed into a projectivity of $\Pi_{\mathbb{L}}$ and a bijection on $\Pi_{\mathbb{L}}$ induced by an automorphism $\zeta$ of $\mathbb{L}$. It depends on the properties of $\zeta$ whether a Plücker transformation $\varphi$ is induced by a projective collineation, a nonprojective collineation or whether $\varphi$ is not induced by any collineation (Theorem 2). In this sense 3-dimensional hyperbolic spaces are exceptional. 
Orthogonality-preserving Plücker transformations of Euclidean spaces have been investigated by W. BenZ and E.M. SchröDER in [1, 2]. Analogously H. HAVLICEK discussed orthogonalitypreserving Plücker transformations of elliptic and symplectic spaces [6, 7]. Without going into details we can conclude that in all these spaces 3-dimensional spaces are exceptional, since orthogonality-preserving Plücker transformations are not necessarily induced by collineations.

\section{PlüCker Spaces And Hyperbolic Spaces}

Let $G$ be an arbitrary non-empty set and $\sim$ a binary relation on $G$ which is symmetric and reflexive. Following [1] we call the structure $(G, \sim)$ a Plücker space, if for each pair $a, b \in G$ there exists a finite number of elements $c_{1}, c_{2}, \ldots, c_{n} \in G$ with

$$
a \sim c_{1} \sim c_{2} \sim \ldots \sim c_{n} \sim b .
$$

A Plücker transformation of $(G, \sim)$ is a bijection $\varphi: G \rightarrow G$ with

$$
a \sim b \Longleftrightarrow a^{\varphi} \sim b^{\varphi} \text { for all } a, b \in G .
$$

All such Plücker transformations form the Plücker group of $(G, \sim)$.

Let $\Pi:=\Pi(\mathcal{P}, \mathcal{G})$ be a Pappian projective space $(2 \leq \operatorname{dim} \Pi:=n<\infty)$ with point set $\mathcal{P}$, line set $\mathcal{G}$ and Euclidean ground field $\mathbb{K}$. The set $\mathcal{H}$ of internal points of an oval quadric $\mathcal{Q}$ in $\Pi$ is non-empty [4, p.54]. Since $\mathbb{K}$ is Euclidean, each projective line $g$ running through a point of $\mathcal{H}$ contains infinitly many points of $\mathcal{H}$ and the section $g \cap \mathcal{Q}$ gives two ideal points. Conversely, each secant of $\mathcal{Q}$ contains an internal point of $\mathcal{Q}\left[4\right.$, p.54]. Now the linear space $\Pi_{h}(\mathcal{H}, \overline{\mathcal{G}})$ with

$$
\overline{\mathcal{G}}:=\{\bar{g}=g \cap \mathcal{H} \mid \bar{g} \neq \varnothing, g \in \mathcal{G}\}
$$

is the Cayley-Klein model of the $n$-dimensional hyperbolic space over $\mathbb{K}$; cf. [5]. We denote the polarity of the absolute quadric $\mathcal{Q}$ by $\pi$. In the following we will distinguish between a secant $g \in \mathcal{G}$ of $\mathcal{Q}$ and the hyperbolic line $\bar{g}:=g \cap \mathcal{H}$. Obviously there is a one-to-one correspondence between secants $g \in \mathcal{G}$ and hyperbolic lines $\bar{g} \in \overline{\mathcal{G}}$.

The polarity $\pi$ gives rise to the following binary relations $\sim$ and $\approx$ on $\overline{\mathcal{G}}$ : For $\bar{a}, \bar{b} \in \overline{\mathcal{G}}$ we put

$$
\begin{array}{ll}
\bar{a} \approx \bar{b}: \Longleftrightarrow a \cap b^{\pi} \neq \varnothing \text { and } \bar{a} \cap \bar{b} \neq \varnothing & \text { (orthogonally intersecting lines) } \\
\bar{a} \sim \bar{b}: \Longleftrightarrow \bar{a} \approx \bar{b} \text { or } a=b & \text { (related lines). }
\end{array}
$$

In [12] there is shown that the structure $(\overline{\mathcal{G}}, \sim)$ forms a Plücker space. Now we investigate the Plücker transformations of this Plücker space in the 3 -dimensional case. Each $\mathcal{Q}$-collineation ${ }^{1}$ induces a Plücker transformation of $(\overline{\mathcal{G}}, \sim)$ [12]. But the question is if all Plücker transformations of $(\overline{\mathcal{G}}, \sim)$ are induced by $\mathcal{Q}$-collineations.

For the investigation of Plücker transformations of $(\overline{\mathcal{G}}, \sim)$ we have to observe first the Plücker space of parallel lines.

\section{The PlüCker Group of $(\overline{\mathcal{G}}, \|)$}

On $\overline{\mathcal{G}}$ there we have the hyperbolic parallelism

$$
\|\subset \overline{\mathcal{G}} \times \overline{\mathcal{G}}: \quad \bar{a}\| \bar{b} \Longleftrightarrow a \cap b \cap \mathcal{Q} \neq \varnothing .
$$

\footnotetext{
${ }^{1} \mathrm{~A} \mathcal{Q}$-collineation is a collineation of $\Pi$ leaving the quadric $\mathcal{Q}$ invariant as a set.
} 
Obviously $\|$ is reflexive, symmetric and for any two lines $\bar{a}, \bar{b}$ we can find a line $\bar{c}$ such that $\bar{a}\|\bar{c}\| \bar{b}$. We infer that $(\overline{\mathcal{G}}, \|)$ is a Plücker space. Now we will show that any bijection $\varphi: \overline{\mathcal{G}} \rightarrow \overline{\mathcal{G}}$ with

$$
\bar{a}\left\|\bar{b} \Longrightarrow \bar{a}^{\varphi}\right\| \bar{b}^{\varphi}, \quad \forall \bar{a}, \bar{b} \in \overline{\mathcal{G}}
$$

is already a Plücker transformation of $(\overline{\mathcal{G}}, \|)$. By abuse of notation, we define $g^{\varphi}$ as the projective line with $\bar{g}^{\varphi} \subset g^{\varphi}$ for all secants $g$ of $\mathcal{Q}$.

If there is given a bijection $\gamma: \mathcal{Q} \rightarrow \mathcal{Q}$ then we are able to define the bijection

$$
\iota(\gamma): \overline{\mathcal{G}} \rightarrow \overline{\mathcal{G}}, \overline{A B} \mapsto \overline{A^{\gamma} B^{\gamma}}(A, B \in \mathcal{Q}, A \neq B) .
$$

For such a $\iota(\gamma)$, parallelism of lines is an invariant property. But since $\gamma$ is a bijection, parallelism of lines is also invariant under $\iota(\gamma)^{-1}$. That means $\iota(\gamma)$ is a Plücker transformation of $(\overline{\mathcal{G}}, \|)$.

Lemma 1. Let $\Pi_{h}$ be a 3-dimensional ${ }^{2}$ hyperbolic space. Any bijection $\varphi: \overline{\mathcal{G}} \rightarrow \overline{\mathcal{G}}$ fulfilling (2) induces a bijection

$$
\gamma: \mathcal{Q} \rightarrow \mathcal{Q}, \quad a \cap b \mapsto a^{\varphi} \cap b^{\varphi} \quad(\bar{a}, \bar{b} \in \overline{\mathcal{G}}, a \cap b \in \mathcal{Q}),
$$

such that $\iota(\gamma)=\varphi$.

Proof. For an $A \in \mathcal{Q}$ let the four distinct lines $\bar{a}, \bar{b}, \bar{c}, \bar{d}$ be elements ${ }^{3}$ of the star $\overline{\mathcal{G}}_{A}$. Their $\varphi$-images have to be again parallel. Assuming that $a^{\varphi}, b^{\varphi}, c^{\varphi}$ are the edges of a triangle $(\subset \mathcal{Q})$ we get the contradiction $a^{\varphi} \cap b^{\varphi}, b^{\varphi} \cap c^{\varphi}, c^{\varphi} \cap a^{\varphi} \in d^{\varphi}$. Therefore all lines $a^{\varphi}, b^{\varphi}, c^{\varphi}, d^{\varphi}$ are running through $A^{\gamma}:=a^{\varphi} \cap b^{\varphi} \in \mathcal{Q}$ and $\gamma: \mathcal{Q} \rightarrow \mathcal{Q}$ is a well-defined mapping. It remains to be shown that $\gamma$ is a bijection.

Assume to the contrary that there exists an $A \in \mathcal{Q}$ without $\gamma$-preimage. Since $\varphi$ is a bijection, we can choose distinct points $B, C \in \mathcal{Q}$ with $A \in B C^{\varphi}$. But $A$ is not an image of $\gamma$ and therefore $B^{\gamma}=C^{\gamma}$. For an arbitrary $D \in \mathcal{Q}$ with $D \neq B, C$ the lines $D B^{\varphi}, D C^{\varphi}$ are distinct, because $\varphi$ is a bijection. So $D^{\gamma}=B^{\gamma}=C^{\gamma}$. But then $\mathcal{Q}^{\gamma}=\left\{B^{\gamma}\right\}$ and $B^{\gamma} \in a^{\varphi}$ for all $\bar{a} \in \overline{\mathcal{G}}$. This contradicts the surjectivity of $\varphi$. We showed the surjectivity and injectivity of $\gamma$ simultaneously, and finally we see that $\iota(\gamma)=\varphi$.

Thus we have

Proposition 1. The Plücker group of $(\overline{\mathcal{G}}, \|)$ consists of all bijections $\varphi: \overline{\mathcal{G}} \rightarrow \overline{\mathcal{G}}$ fulfilling (2).

Proposition 1 will help us to show an analogous theorem in the Plücker space $(\overline{\mathcal{G}}, \sim)$.

\section{The PlüCker Group of $(\overline{\mathcal{G}}, \sim)$}

The following two Lemmata will be helpful in the examination of Plücker transformations of $(\overline{\mathcal{G}}, \sim)$.

Lemma 2. Let $\bar{a}$ be an arbitrary hyperbolic line and $\overline{\mathcal{G}}_{A}$ a star of parallel lines with $\bar{a} \notin \overline{\mathcal{G}}_{A}$. Then there exists a unique line $\bar{g} \in \overline{\mathcal{G}}_{A}$ with $\bar{a} \sim \bar{g}$.

Proof. This unique line is $\bar{g}=\overline{\left(\left(A^{\pi} \cap a\right)^{\pi} \cap a\right) A}$.

\footnotetext{
${ }^{2}$ Lemma 1 is true for any $n$-dimensional hyperbolic space $(2 \leq n<\infty)$, but in this paper we focus onto the 3-dimensional case.

${ }^{3}$ Since char $\mathbb{K}=0$ any star contains 4 distinct lines.
} 
Lemma 3. In 3-dimensional hyperbolic spaces there is:

$$
\bar{a} \neq \bar{b} \text { are parallel } \Longleftrightarrow \nexists \bar{n} \in \overline{\mathcal{G}} \text { with } \bar{a} \sim \bar{n} \sim \bar{b} .
$$

Proof. From [12] (Theorem 1 and Lemma 1) we already know that hyperparallel and skew lines have a common orthogonal line. Since $\operatorname{dim} \Pi_{h}=3$ the subspace $(a \vee b)^{\pi} \neq \varnothing$, if $\bar{a} \cap \bar{b} \neq \varnothing$. Therefore $\bar{n}$, with $n=(a \vee b)^{\pi} \vee(\bar{a} \cap \bar{b})$, intersects $\bar{a}$ and $\bar{b}$ orthogonally.

Assume to the contrary that two parallel lines $\bar{a} \neq \bar{b}$ do have a common orthogonal line $\bar{n}$. Then $a, b$ intersect in the external point $n^{\pi} \cap(a \vee b)$. This is a contradiction.

Now we are able to show:

Proposition 2. Let $\Pi_{h}$ be a 3-dimensional hyperbolic space with line set $\overline{\mathcal{G}}$. Then for each bijection $\varphi: \overline{\mathcal{G}} \rightarrow \overline{\mathcal{G}}$ with

$$
\bar{a} \sim \bar{b} \Longrightarrow \bar{a}^{\varphi} \sim \bar{b}^{\varphi}, \quad \forall \bar{a}, \bar{b} \in \overline{\mathcal{G}}
$$

there exists a bijection $\gamma$ of $\mathcal{Q}$ such that $\iota(\gamma)=\varphi$. Moreover, $\varphi$ is a Plücker transformation of $(\overline{\mathcal{G}}, \sim)$.

Proof. Two distinct parallel lines $\bar{a}^{\varphi}, \bar{b}^{\varphi}$ have no common orthogonal line (see Lemma 3). Since orthogonal intersection is an invariant property under $\varphi$,

$$
\bar{a}^{\varphi}\left\|\bar{b}^{\varphi} \Longrightarrow \bar{a}\right\| \bar{b}, \quad \forall \bar{a}, \bar{b} \in \overline{\mathcal{G}} .
$$

By Proposition 1, we know that $\varphi$ is a Plücker transformation of $(\overline{\mathcal{G}}, \|)$ and therefore

$$
\bar{a}\left\|\bar{b} \Longrightarrow \bar{a}^{\varphi}\right\| \bar{b}^{\varphi}, \quad \forall \bar{a}, \bar{b} \in \overline{\mathcal{G}} .
$$

So, according to Lemma $1, \varphi$ gives rise to a bijection $\gamma: \mathcal{Q} \rightarrow \mathcal{Q}$ with $\iota(\gamma)=\varphi$.

Now let $\bar{a} \nsim \bar{b}$ and $A \in \mathcal{Q}$ with $b \not \ngtr A \in a$. By Lemma 2, there exists exactly one $n \in \mathcal{G}$ with $A \in n$ and $\bar{n} \approx \bar{b}$. Moreover, $\bar{n}^{\varphi} \approx \bar{b}^{\varphi}$ and $n^{\varphi} \ni A^{\gamma} \in a^{\varphi}$. By $\bar{a} \neq \bar{n}$, we get $\bar{a}^{\varphi} \neq \bar{n}^{\varphi}$ and finally $\bar{a}^{\varphi} \not \bar{b}^{\varphi}$.

\section{Analytic Description of the Plücker Group of $(\overline{\mathcal{G}}, \sim)$}

We already know that a Plücker transformation induces a bijection on the absolute quadric $\mathcal{Q}$. Furthermore we show that there exists a bijection between the point set of $\mathcal{Q}$ and the projective line $\Pi_{\mathbb{L}}$ over the unique quadratic extension $\mathbb{L}:=\mathbb{K}(i)$ of $\mathbb{K}$ with $i^{2}=-1$. Then we are able to show that exactly the harmonic mappings in $\Pi_{\mathbb{L}}$ are induced by Plücker transformations of $(\overline{\mathcal{G}}, \sim)$; cf.Theorem 1.

So let us introduce the bijection between $\mathcal{Q}$ and $\Pi_{\mathbb{L}}$. The projective space $\Pi$ is isomorphic to the projective space $\Pi\left(\mathbb{K}^{4}\right)$ over the vector space $\mathbb{K}^{4}$. As $\mathbb{K}$ is a Euclidean field, it can be ordered uniquely. For any $a \in \mathbb{K}^{+}$there exists an element $\sqrt{a} \in \mathbb{K}$ with $(\sqrt{a})^{2}=a$. Therefore [13, p. 234ff.] the quadric $\mathcal{Q}$ has the same normal form as in real projective spaces. We can describe $\mathcal{Q}$ by the equation

$$
-x_{0} x_{3}+x_{1}^{2}+x_{2}^{2}=0 .
$$

Now we are able to define the bijection $\xi$ between the projective line $\Pi_{\mathbb{L}}$ and $\mathcal{Q}$ (see [9, p.814]):

$$
\xi: \Pi_{\mathbb{L}} \rightarrow \mathcal{Q}, \quad \mathbb{L}\left(x_{0}+i x_{1}, y_{0}+i y_{1}\right) \mapsto \mathbb{K}\left(x_{0}^{2}+x_{1}^{2}, x_{0} y_{0}+x_{1} y_{1}, x_{0} y_{1}-x_{1} y_{0}, y_{0}^{2}+y_{1}^{2}\right) .
$$


Let $\beta: \Pi_{\mathbb{L}} \rightarrow \Pi_{\mathbb{L}}$ be a bijection of $\Pi_{\mathbb{L}}$. Then by $\beta$ there is induced the bijection $\xi^{-1} \beta \xi$ on $\mathcal{Q}$. Now we have to find out under which conditions such a bijection induces a Plücker transformation $\iota\left(\xi^{-1} \beta \xi\right)$ of $(\overline{\mathcal{G}}, \sim)$.

If there is given a projectivity $\alpha$ of $\Pi_{\mathbb{L}}$, then the bijection $\xi^{-1} \alpha \xi$ of $\mathcal{Q}$ can be extended to a projective $\mathcal{Q}$-collineation [9, p.815]. Therefore $\iota\left(\xi^{-1} \alpha \xi\right)$ is a Plücker transformation of $(\overline{\mathcal{G}}, \sim)$.

Proposition 3. Let $A, B, C, D \in \mathcal{Q}$ be four distinct points. Then $\overline{A B}$ and $\overline{C D}$ intersect orthogonally if and only if $\left(A^{\xi^{-1}}, B^{\xi^{-1}}\right)$ and $\left(C^{\xi^{-1}}, D^{\xi^{-1}}\right)$ are harmonic pairs.

Proof. By the action of the group of projectivities of $\Pi_{\mathbb{L}}$ or equivalently, by the action of the induced group of $\mathcal{Q}$-collineations, we may assume w.l.o.g. that

$$
A^{\xi^{-1}}=\mathbb{L}(1,0), B^{\xi^{-1}}=\mathbb{L}(0,1), C^{\xi^{-1}}=\mathbb{L}(1,1)
$$

or equivalently,

$$
A=\mathbb{K}(1,0,0,0), B=\mathbb{K}(0,0,0,1), C=\mathbb{K}(1,1,0,1) .
$$

If the pairs $\left(A^{\xi^{-1}}, B^{\xi^{-1}}\right),\left(C^{\xi^{-1}}, D^{\xi^{-1}}\right)$ are harmonic then

$$
D^{\xi^{-1}}=\mathbb{L}(1,-1) \text { and } D=\mathbb{K}(1,-1,0,1) .
$$

It is easy to prove that $\overline{A B}, \overline{C D}$ intersect orthogonally. Vice versa we have given $\overline{A B}, \overline{C D}$ intersecting orthogonally. We may assume that the coordinates of $A, B, C$ are the ones from above. With Lemma 2 we get that also $D$ has the coordinates from above. But we already know that the $\xi$-preimages of $(A, B),(C, D)$ are harmonic pairs.

Now it is obvious that every bijection on $\Pi_{\mathbb{L}}$ preserving the harmonic position of pairs induces a Plücker transformation of $(\overline{\mathcal{G}}, \sim)$. Again we see that every projectivity $\alpha$ of $\Pi_{\mathbb{L}}$ yields the Plücker transformation $\iota\left(\xi^{-1} \alpha \xi\right)$ of $(\overline{\mathcal{G}}, \sim)$. By each automorphism $\zeta: \mathbb{L} \rightarrow \mathbb{L}$ we have given the bijection

$$
\bar{\zeta}: \Pi_{\mathbb{L}} \rightarrow \Pi_{\mathbb{L}}, \quad \mathbb{L}\left(x_{0}+i x_{1}, y_{0}+i y_{1}\right) \mapsto \mathbb{L}\left(\left(x_{0}+i x_{1}\right)^{\zeta},\left(y_{0}+i y_{1}\right)^{\zeta}\right),
$$

which preserves also the harmonic position of pairs ([4, p.40]). Therefore the Plücker transformation $\iota\left(\xi^{-1} \bar{\zeta} \xi\right)$ is induced by $\zeta$.

Theorem 1. Let $\Pi_{h}$ be a 3-dimensional hyperbolic space and $\varphi$ a Plücker transformation of $(\overline{\mathcal{G}}, \sim)$. Then $\varphi$ can be decomposed into a product $\varphi=\iota\left(\xi^{-1} \alpha \xi\right) \iota\left(\xi^{-1} \bar{\zeta} \xi\right)$ of two Plücker transformations where:

1. $\zeta$ is an automorphism of the quadratic extension $\mathbb{K}(i):=\mathbb{L}$ of $\mathbb{K}$ with $i^{2}=-1$.

2. $\alpha$ is a projectivity of $\Pi_{\mathbb{L}}$, the projective line over $\mathbb{L}$.

Moreover $\varphi$ is induced by a harmonic mapping ${ }^{4}$ of $\Pi_{\mathbb{L}}$.

Proof. From above we know that a Plücker transformation $\varphi$ induces a bijection $\gamma$ on $\mathcal{Q}$. Furthermore we have induced a bijection on $\Pi_{\mathbb{L}}$. Orthogonal intersection is invariant under $\varphi$. Therefore the harmonic position of pairs in $\Pi_{\mathbb{L}}$ is invariant under $\beta:=\xi \gamma \xi^{-1}$ (Proposition 3). Now we decompose the harmonic mapping $\beta$.

Obviously each projectivity of $\Pi_{\mathbb{L}}$ is a harmonic mapping. Since through a triple of points and it's image a projectivity $\alpha$ is given [3, p.142], we may assume that the product $\beta \alpha$ has three fixed points. So we can use the Theorem of StaudT-HuA [4, p. 40] and get that $\beta \alpha=\bar{\zeta}$, for an automorphism $\zeta: \mathbb{L} \rightarrow \mathbb{L}$.

\footnotetext{
${ }^{4} \mathrm{~A}$ harmonic mapping of $\Pi_{\mathbb{L}}$ is a bijection on $\Pi_{\mathbb{L}}$ which maps harmonic pairs onto harmonic pairs.
} 


\section{PlüCker Transformations and Collineations}

Theorem 1 gives no answer to the question whether all Plücker transformations of $\Pi_{h}$ are induced by collineations of $\Pi$ or not. But by Theorem 1 we just have to investigate Plücker transformations $\varphi$ that are induced by

1. projectivities of $\Pi_{\mathbb{L}}$

2. automorphisms $\zeta$ of $\mathbb{L}$.

In the following we will use the notation introduced in section 5. Two collineations $\psi_{1}, \psi_{2}$ fixing the quadric $\mathcal{Q}$ are identical if $\psi_{1}\left|\mathcal{Q}=\psi_{2}\right| \mathcal{Q}$. This is obvious because every point of $\Pi$ is the intersection point of two secants of $\mathcal{Q}$. Therefore, if there exists a collineation of $\Pi$ inducing $\varphi$, it is already unique.

We already know that projectivities of $\Pi_{\mathbb{L}}$ induce projective $\mathcal{Q}$-collineation $[9$, p.815]. Therefore Plücker transformations induced by automorphisms of $\mathbb{L}$ remain to be investigated in detail.

On $\Pi_{\mathbb{L}}$ the automorphism $\zeta$ induces the bijection $\bar{\zeta}$ (see (4)).

1. In case $\mathbb{K}^{\zeta}=\mathbb{K}$ we get for $\mathbb{L}=\mathbb{K}(i)$ that $i^{\zeta}= \pm i$ [13, p.277]. Furthermore, $\bar{\zeta}$ yields (with respect to $[9, \mathrm{p} .814],(3))$ the bijection $\xi^{-1} \bar{\zeta} \xi$ on $\mathcal{Q}$, which is obviously also induced by the collineation

$$
\psi_{\zeta}: \Pi \rightarrow \Pi, \mathbb{K}\left(a_{0}, a_{1}, a_{2}, a_{3}\right) \mapsto \mathbb{K}\left(a_{0}^{\zeta}, a_{1}^{\zeta}, \pm a_{2}^{\zeta}, a_{3}^{\zeta}\right)
$$

If $\zeta \mid \mathbb{K}=\operatorname{id} d_{\mathbb{K}}$ then $\psi_{\zeta}$ is a projective collineation, otherwise $\zeta \mid \mathbb{K} \neq \mathrm{id} \mathbb{K}$ and $\psi_{\zeta}$ is a nonprojective collineation.

2. Now let $\zeta$ be an automorphism with $\mathbb{K}^{\zeta} \not \subset \mathbb{K}$. That means there exists an element $x \in \mathbb{K}$ with $x^{\zeta} \notin \mathbb{K}$. The points $A=\mathbb{L}(1,0), B=\mathbb{L}(0,1), C=\mathbb{L}(1,1)$ are fixed under $\bar{\zeta}$, but not $D=\mathbb{L}(1, x)$. The $\xi$-images of $A, B, C, D$ are on a planar section of $\mathcal{Q}$. Since $x^{\zeta} \notin \mathbb{K}$, the $\xi$-images of $A^{\bar{\zeta}}, B^{\bar{\zeta}}, C^{\bar{\zeta}}, D^{\bar{\zeta}}$ are not coplanar. Therefore the bijection $\xi^{-1} \bar{\zeta} \xi$ on $\mathcal{Q}$ cannot be extended to a collineation of $\Pi$. The Plücker transformation $\iota\left(\xi^{-1} \bar{\zeta} \xi\right)$ is not induced by a collineation.

We remark that under each $\mathcal{Q}$-collineation hyperbolic distances [11, p.158] are transformed by the accompanying automorphism of $\mathbb{K}$. Therefore each projective $\mathcal{Q}$-collineation preserves hyperbolic distances, so it is a hyperbolic motion.

The real numbers $\mathbb{R}$ form an Euclidean field. Their unique quadratic extension is $\mathbb{C}$. But the existence of automorphisms $\zeta: \mathbb{C} \rightarrow \mathbb{C}$ with $\mathbb{R}^{\zeta} \not \subset \mathbb{R}$ is well known (see [10, p.517] or [1, p.114f]). Therefore in real hyperbolic 3-spaces there exist orthogonality-preserving Plücker transformations that are not induced by collineations of the ambient projective space. Such Plücker transformations do not exist in hyperbolic spaces with dimension other than three [12]. Summing up Proposition 2, Theorem 1, and the results from above we get

Theorem 2. Let $\Pi_{h}$ be a 3-dimensional hyperbolic space and $\varphi: \overline{\mathcal{G}} \rightarrow \overline{\mathcal{G}}$ a bijection with

$$
\bar{a} \sim \bar{b} \Longrightarrow \bar{a}^{\varphi} \sim \bar{b}^{\varphi} \quad \forall \bar{a}, \bar{b} \in \overline{\mathcal{G}} .
$$

Then $\varphi=\iota\left(\xi^{-1} \alpha \xi\right) \iota\left(\xi^{-1} \bar{\zeta} \xi\right)$ is a Plücker transformation, where $\alpha$ is a projectivity of $\Pi_{\mathbb{L}}$ and $\zeta$ is an automorphism of $\mathbb{L}$. Moreover: 
1. $\varphi$ is induced by a projective $\mathcal{Q}$-collineation of the ambient projective space, if $\zeta \mid \mathbb{K}=\mathrm{id}{ }_{\mathbb{K}}$.

2. $\varphi$ is induced by a non-projective $\mathcal{Q}$-collineation of the ambient projective space, if $\mathbb{K}^{\zeta}=\mathbb{K}$ but $\zeta \mid \mathbb{K} \neq \mathrm{id}_{\mathbb{K}}$.

3. $\varphi$ is not induced by a collineation, if $\mathbb{K}^{\zeta} \not \subset \mathbb{K}$.

\section{REFERENCES}

[1] Benz W.: Geometrische Transformationen, B.I. Wissenschaftsverlag, Mannheim Leipzig Zürich, 1992.

[2] Benz W., SchröDer E.M.: Bestimmung der orthogonalitätstreuen Permutationen euklidischer Räume, Geom. Dedicata 21 (1986), 265 - 276.

[3] Brauner H.: Geometrie projektiver Räume I, B.I. Wissenschaftsverlag, Mannheim Wien Zürich, 1976.

[4] Brauner H.: Geometrie projektiver Räume II, B.I. Wissenschaftsverlag, Mannheim Wien Zürich, 1976.

[5] Giening O.: Vorlesungen über höhere Geometrie, Vieweg, Braunschweig/ Wiesbaden, 1982.

[6] Havlicek H.: On Plücker Transformations of Generalized Elliptic Spaces, Rend. Mat. Appl., VII Ser. 14 (1994), 39 - 56.

[7] Havlicek H.: Symplectic Plücker Transformations, Math. Pannonica 6 (1995), 145 - 153.

[8] HavliceK H.: A Characteristic Property of Elliptic Plücker Transformations, J. Geom. 58 (1997), $106-116$.

[9] Herzer A.: Chain Geometries, Buekenhout F. (ed.), Handbook of Incidence Geometry, Elsevier Science, 1995.

[10] Jacobson N.: Basic Algebra II, W.H. Freeman and Company, New York, 1989.

[11] Karzel H., Kroll H.-J.: Geschichte der Geometrie seit Hilbert, Wiss. Buchges., Darmstadt, 1988.

[12] List K.: On Orthogonality-preserving Plücker Transformations of Hyperbolic Spaces, Abh. Math. Sem. Univ. Hamburg, to appear.

[13] SchaAl H.: Lineare Algebra und Analytische Geometrie, Band II, Vieweg-Verlag, Braunschweig, 1976.

TU Wien - Institut für Geometrie

Wiedner Hauptstraße 8-10

A-1040 Wien

klaus.list@tuwien.ac.at

Eingegangen am 30. Juli 1999; in revidierter Fassung am 25. November 1999 